\title{
The Role of M1/M2 Transition of the Brain Macrophages in Alzheimer's Disease
}

\author{
Neha Chitre ${ }^{1}$,PriyaGhumatkar ${ }^{2}$, Dr. Sadhana Sathaye ${ }^{3}$ \\ ${ }^{I}$ (Department of Pharmaceutical Sciences and Technology, Institute of Chemical Technology, India) \\ ${ }^{2}$ (Department of Pharmaceutical Sciences and Technology, Institute of Chemical Technology, India) \\ ${ }^{3}$ (Department of Pharmaceutical Sciences and Technology, Institute of Chemical Technology, India)
}

\begin{abstract}
Alzheimer's disease (AD) is a neurodegenerative disorder that is characterized by the presence of neurofibrillary tangles (NFT) and senile plaques caused due to amyloid beta (Aß) aggregation in the brain. Macrophage like resident immune cells in the brain called microglia play a pivotal role in the CNS immunity. They are constantly engaged in removing damaged cells which is a very important part of maintaining homeostasis. Microglial cells contribute to the clearance of AB by the process of phagocytosis, which may lead to delaying the progression of $A D$. Microglial cells also exhibit their neuroprotective role by secreting various growth factors. However, hyper activation of microglia leads to a sustained overproduction of microglial proinflammatory mediators which are neurotoxic and which raise great concern that systemic inflammation can exacerbate or possibly trigger neurological diseases. The aim of the present study is to unravel the dual role of microglia by studying the macrophage M1/M2 Polarization and to further exploit this link in implicating newer therapeutic interventions.
\end{abstract}

Keywords -Alzheimer's disease, amyloid- $\beta$, inflammation, immunity, microglia

\section{Introduction}

Alzheimer's disease (AD) is a progressive neurodegenerative disorder, and the most frequent cause of dementia in the elderly population .It ischaracterized by two major pathological hallmarks-Neurofibrillary tangles and numerous neuritic plaques which lead to synaptic dysfunction and eventually cause neuronal death. This results in the decline of memory, loss of language skills and delusion in the final stages. The symptoms generally start gradually and are dismissed as regular symptoms of old age. The Central Nervous immune system plays a very vital role in delaying the progression of Alzheimer's disease. There are numerous cell types that act in accord to maintain the integrity of CNS and its functions. They are usually broadly classified as the neurons, endothelial cells and glial cells. Microglia are the most characteristic cells of CNS innate immunity.The microglial cells are macrophages that perform an immune activity of scavenging dead cells and debris which is the vital element of the brain immune surveillance. Alzheimer's disease is caused mainly by the deposition of $A \beta$ into plaques in the CNS. Microglial cells help in clearing the $A \beta$ plaques through the process of phagocytosis and thus serve as potential therapeutic targets. These macrophages are extremely sensitive to their external environment and can acquire two different phenotypes on thebasis of this stimulus. The pro inflammatory phenotype (M1) is associated with the neurotoxic role of microglia and is induced by lipopolysaccharide stimulation. The anti- inflammatory phenotype (M2) is associated with the neuroprotective roles carried by the microglial cells and is induced by interleukin-4/10 (IL4/10) stimulation [1]. Thus, this transition between the M1/M2 polarization states is of great importance and presents the scope of manipulation of the macrophage phenotypes in order to promote neuroprotective functions. One of the strategies that is being explored on these lines is Immunotherapy that aims at promoting the phagocytosis of $A \beta$ plaques from the brains of $\mathrm{AD}$ patients by the microglial cells. The aim of this study is to examine the two activation states of the macrophages and then asses the functional relevance of this process for future prospects.

\section{Innate Immunity in The CNS}

The neurons, glial cells and the endothelial cells act in coordination and form a coherent view of the CNS as a whole, as opposed to the idea of being a blend of compartmentalized cell types. [2] Microglia are the typical and most important cells of CNS innate immunity.These macrophages perform a scavenging activity and help in maintaining a state of homeostasis in the brain environment. The endothelial cells act as mediators of immune processes and form a link between the peripheral immune responses and the CNS. The Blood Brain is a membrane that is extremely tightly regulated and plays the key role in controlling in innate immune responses by fulfilling two major functions:

1. It acts as a physical barrier by separating the CNS from the circulation due to the membrane formed by the endothelial cells which are attached to each other tightly due to the tight junctions and adherent junctions.[3] 
2. Secondly, it acts as a selective exchange barrier that helps in the transport of certain molecules and proteins via various processes and transporter molecules.

The astrocytes are another important aspect of the CNS Immunity and they have endfeet which associate with the endothelial cells that form the Blood Brain Barrier. They also produce and secrete numerous bioactive molecules that modify endothelial function, such as TNFa, IL-1b, IL-3, IL-6.The astrocytes also act as scaffold cells by guiding neurons during development. [4.]

\subsection{Innate Immune Responses in the CNS}

The CNS engages in a multiplex immune response when stimulated due to any mechanical injury, trauma or stress which could be due to the onset of pathogenic infections, or the accumulation of toxins. During pathological conditions, the CNS is stimulated with small protein patterns called pathogen associated molecular patterns (PAMPS) and danger associated molecular patterns (DAMPS) that on detection by receptors initiate specific immune responses. Such patterns consist of proteins from the bacterial membranes such as peptidoglycans, intracellular proteins and non-protein products such as ATP .The proteins are recognized by pattern recognition receptors (PRRs) mainly belonging to the major classes of Toll-like receptors (TLRs), Nodlike receptors (NLRs), and RIG1-like receptors (RLRs).The activation of such receptors causes specific pathways to get induced which in turn promotes the release of specific cytokines that play a role in resolving the injuries that have occurred.

\subsection{Innate Immunity in Alzheimer's disease}

Alzheimer's disease is mainly characterised by the presence of $A \beta$ plaques and neurofibrillary tangles in the CNS. The innate immune system contributes to the development of the pathology of AD. Chronic exposure of microglia to $\mathrm{A} \beta$ contributes to uncontrolled inflammation and the release of toxic free radicals and reactive oxygen species which greatly increases the oxidative stress. Whether the activation of the innate immune system is a cause or a consequence of the development of $\mathrm{AD}$ is still highly debated. In vitro studies have shown that the fibrilary tangles activate microglia that leads to the release of TNF $\alpha$ leading to neuronal death. The microglial cells then enter a non-resolving chronic inflammatory circle of continuous inefficient activation, thus contributing to disease progression. However, the innate immune system also plays a role in the clearance of the Amyloid Beta Plaques and can play a beneficial role in AD. This would explain the detrimental effects of completely suppressing the innate immune response, while beneficial effects of inhibiting selective parts of it can prove to be an efficient therapeutic strategy.

\section{Microglia in Alzheimer's Disease: A Multifaceted Relationship}

The concept of neuroinflammation emerged when several soluble inflammatory markers were identified in brains of Alzheimer's patients. [5].Some reports showed that $A \beta$ triggered microglial cell activation causing the production of pro-inflammatory cytokines and neurotoxic molecules which then accelerated the neurodegenerative disorder. In healthy conditions, microglial cells have a ramified and are characterized by numerous branching processes. Under normal physiology, the macrophages are involved in numerous processes like clearance of dying cells and eliminating excess axons. They also release a variety of cell signalling factors like neurotrophins. Multiple pathologic events like bacterial infections or damage can lead to the activation of microglia rapidly. This activation causes a transition in the microglial phenotype and can lead to the production of several pro inflammatory cytokines and neurotoxic molecules. However, the macrophages also help in phagocytosis of the $\mathrm{A} \beta$ plaques and exhibit neuroprotective roles, thus exerting a biphasic role.

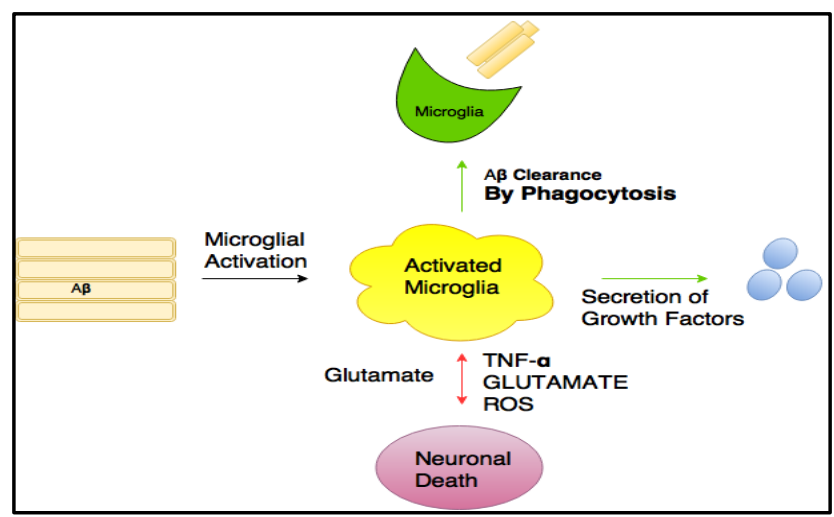

Fig. 1. The Dual Role of Microglia : Neuroprotective Role of $A \beta$ clearance and secretion of growth factors and Neurotoxic Role of exacerbating neuronalcell death due to the release of excessive TNF- $\alpha$ and reactive oxygen species. 


\subsection{The physiology and functioning of microglial cells}

The resident microglia are mononuclear phagocytes and constitute the main resident immune cell population of the brain, representing up to $10 \%$ of the cells in the brain [6]. It is most widely accepted that the microglia originate from mesoderm bone marrow precursor cells in embryonic period. Contrary to the previous view that the microglial cells are in a "resting state" under normal physiological conditions, research has now shown that these cells are never resting and are continuously patrolling the brain in search of any imbalance in cerebral homeostasis. Recent findings haves shown that microglial cells scan the microenvironment by making use of their dynamic cellular processes as sentinels and detect even a slight change occurring in brain homeostasis [7]. Microglial cells rapidly get activated in presence of a threat and adopt a less ramified phenotype which is characterized with a large soma thereby forming an amoeboid shape. On activation, these cells respond in order to deal with the detected threat and respond through various means like phagocytosis or the release of various molecular mediators. The dynamic nature of microglia translates their complex roles as regulators and modulators of neuronal function under physiological conditions [8]. In the healthy brain, there is great evidence which suggests that microglia support neuronal function via two major mechanisms, phagocytosis and biochemical interactions. Several studies have highlighted the role of microglia in maintaining the biochemical neuronal function and their contribution towards maintenance of synaptic plasticity. Microgliahave been shown to contribute to the regulation of neurotransmission in collaboration with astrocytes [9].

\section{The Microglial Activity Switch}

The microglial cells are always in an active state, and are constantly surveying the microenvironment of the brain. When the term "activated" is used specifically, it outlines a shift in the activity of the cells to carrying out a specific immune function from surveying the microenvironment. It is suggested that there are two different microglial phenotypes which are based on the M1/M2 activation states of the macrophages. M1 microglia are characterized by the excessive release of several pro-inflammatory cytokines, such as tumor necrosis factor- $\alpha(\mathrm{TNF} \alpha)$ and interleukins like IL1 $\beta$, and IL6, and have a prominent phagocytic capacity [10]. When the two signals of interferon $-\Upsilon$ and tumor necrosis factor combine, it promotes the Microglial M1 Phenotype which exhibits an enhanced capacity of being tumoricidal. Thus, the M1 Phenotype is produced as a result of a cell-mediated immune response to conditions of stress or on the onset of viral infections. On the other hand, the M2 microglia are characterized by the secretion of growth and neurotropic factors, such as transforming growth factor- $\beta$ (TGF $\beta$ ) and Interleukin-10 (IL-10).The M2 Phenotype is developed as a response to wounds or injury, in an attempt to promote wound healing. Thus this phenotype is characterized by the secretion of growth factors like IL-4 that play a role in producing extracellular matrix by causing the stimulation of arginase activity in macrophages which causes the conversion of arginine to ornithine, a collagen precursor. It has been proposed that a shift towards a M1 phenotype exacerbates the inflammatory response, whereas tissue repair and healing is promoted when a shift towards M2 Phenotype takes place.[11].Recent studies from macrophages suggested that the same cell has the potential and ability to acquire M1 or M2 phenotype based on either the nature of the stimuli and/or on the original activation status of the cell before it had any exposure to the stimuli [12].

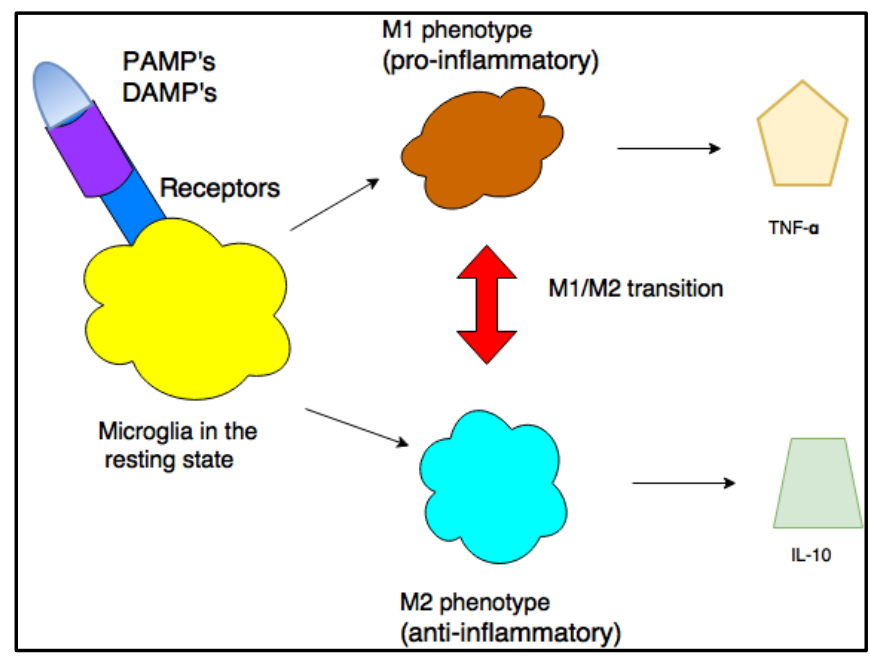

Fig. 2: The M1/M2 Polarization of the microglial cells as a response to stimulationby Pathogen Associated Molecular Patterns (PAMP's) and Danger Associated Molecular Patterns (DAMP's) characterized by the release of pro-inflammatory Factors like TNF- $\alpha$ and anti - inflammatory factors like IL-10. 


\section{Mechanisms of $\mathbf{A} \square$-Induced Microglial Activation}

$\mathrm{A} \beta$ has the ability to bind to microglia and can thus activate them by interaction with pattern recognition receptors (PRRS). Microglia express many PRRs, which recognize and bind to both PAMPs or danger-associated molecular patterns (DAMPs) such as $A \beta$.Interaction of microglia with $A \beta$ provokes their inflammatory actions via PRR's.

There are various receptors that are involved in the recognition of $\mathrm{A} \beta$ and the subsequent microglial activation.

1. Toll like receptors are a type-1 integral glycoproteins. [13]. Studies have demonstrated that the response of microglial cells to the fibrillar forms of $\mathrm{A} \beta$ require the participation of Toll like Receptors and the coreceptors CD14. [14].

2. Receptor for advanced end glycation products (RAGE) is a member of the immunoglobulin superfamily of cell proteins and is reported to mediate the pro inflammatory effects of $A \beta$ by activating the MAPkinase p38 pathway which lead to an enhanced cytokines production and caused an exacerbation of the inflammatory response which further causes the recruitment of microglial cells for phagocytosis. [15].

3. Various scavenger receptors (SR) like SR-A are receptors for insoluble fibrillar aggregates of amyloid betal and are expressed by the activated microglial cells.

4. $\mathrm{A} \beta$ can also bind to members of the seven-transmembrane $\mathrm{G}$ protein coupled receptors known as formyl peptide receptors (FPR), FPR-like 1 (FPRL-1), and FPR-like 2 [16]. FPRs are expressed on several immune cells like leukocytes, monocytes, and microglia.

5. One of the categories of cell surface molecules on microglia that are upregulated when these cells get activated are the Complement receptors [17]. A $\beta$-induced complement activation leads to generation of $\mathrm{C} 1 \mathrm{q}, \mathrm{C} 4$, and $\mathrm{C} 3$ activation fragments around the plaques. Microglial cells then express complement proteins which increases the phagocytic uptake.

\section{Neuroprotective Role of Microglia}

It is commonly believed that the initial microglial activation in Alzheimer's disease can promote the clearance of $A \beta$ plaques and can thus contribute to delaying the disease progression if senile plaque formation can be prevented. Thus it is reported that the activation of microglia is beneficial and protective in the initial stages of the disease. [18]. Microglial cells can help in the attenuation of the A $\beta$ toxicity by promoting the phagocytosis of $\mathrm{A} \beta$ and can exhibit neuroprotective properties by releasing antioxidants and neurotrophic factors. The process of phagocytosis is associated with the inflammatory reactions that occur during the uptake of microbes or other molecules via TLRs and Fc receptors, while phagocytosis of apoptotic cells is executed without inflammation via phosphatidylserine receptors such as T-cell-immunoglobulin-mucin-4 (TIM-4). If inflammatory response occurs in great amounts, it can be toxic to the neurons as excessive. The microglia can be involved in Amyloid Beta clearance either directly via phagocytosis or indirectly via the secretion of enzymes that promote the clearance. Recent literature, has demonstrated more many functions ofmicroglial cells that extend beyond the primary function of immune surveillance. Microglia actively participate in plasticity and maintenance of the adult CNS by secreting cytokines and neurotrophic factors including BDNF [19] They also play a role in refining the neuronal circuit by pruning synapses and axonal terminals [20]. There are more neurons that are produced than required for the development of cerebral cortex during foetal neurogenesis. Thus, in attempt to maintain conditions of equilibrium and homeostasis, the over production of neurons by microglia is limited by phagocytizing neural precursor cells as neurogenesis nears completion. During the natural surveillance activities, microglial cells express surface molecules and secrete factors that affect neuron and astrocyte function. In the healthy brain conditions, microglial cells display a "homeostatic" phenotype, while continuously monitoring the surrounding environment .They play an active role in synaptic pruning and cause the expression of surface molecules which influence astrocytes and neuron function and promote the clearance of cellular debris. Thus, they perform a wide range of activities that support maintenance of homeostasis in the brain microenvironment [21]. The microglial cells also play a role in the embryonic fore brain where they regulate the outgrowth of dopaminergic axons in the forebrain and shape the fasciculation of axonal tracts in the corpus callosum by providing trophic support to neurite formation in the pre natal CNS. [22]Microglia are also known to supress apoptosis and increase proliferation of neural stem cells by producing Insulin-like growth factor-1 which promotes injury -induced neurogenesis. [23].

\section{Neurotoxic Role of Microglia}

Traditionally, the role of microglia was associated with that of a passive by stander when it came to neuronal death. However, ongoing research has now shown that the response of microglial cells to the dying neurons can be detrimental to neuronal survival and can actually exacerbate the death of neurons. Microglia can play a central role in the inflammatory component of Alzheimer's disease. The molecules that are released by the dying neurons can further propel the microglial activation and induce more toxicity. The oligomeric as well 
as fibrillarforms of amyloid beta have been shown to stimulate the microglial cells to secrete pro inflammatory molecules like tumor necrosis factor, macrophages inflammatory protein 1- (MIP-1) and free radicals like super oxides. This causes oxidative damage to proteins, lipids and DNA. The expression of NADPH oxidase, a multi subunit enzyme complex responsible for the production of ROS, is upregulated in AD [24]. The release of a large amount of glutamate by activated microglia through upregulation of glutaminase expression which induces excitoneurotoxicity through NMDA receptor signalling.[25].Excessive glutamate causes an increase in the $\mathrm{Ca} 2+$ influx via the NMDA receptor leading to $\mathrm{Ca} 2+$ /calmodulin-dependent protein kinase (CaMK) activation. The nitric oxide that is induced by CaMK inhibits mitochondrial function Neuritic beading is the formation of bead like structures in the dendrites and axons and a neuropathological sign that is a feature of neuronal cell dysfunction in various diseases including AD. It was reported that the excessive glutamate that is released from activated microglia induces neuritic beading by causing the impairment of dendritic and axonal transport through NMDA receptor signalling [26].Dopaminergic neurons are inherently susceptible to the harmful effects of microglial activation. One hypothesis regarding this is the generation of oxidative insult from microglia. The low intracellular glutathione in the dopamine neurons renders them more susceptible to the oxidative stress caused due to microglial activation relative to other cells. Therefore as amyloid beta is itself toxic to neurons, it is of great importance to study the neurotoxicity additionally induced by microglia in Alzheimer's disease.

\section{Future Prospects and Scope for Therapeutic Intervention}

One of the strategies that have been developed to clear $A \beta$ plaques from the brains of $A D$ patients is immunotherapy. A great number of vaccines and monoclonal antibodies have been tested in clinical trials. .Therapeutic vaccination with $A \beta$ antibodies in mice evidenced the $\mathrm{Fc}$-mediated uptake and clearance of $\mathrm{A} \beta$ antibody complexes by local activated microglia [27]. It was thus proposed that microglial activation by active immunization can be a valid mechanism for clearance of senile plaques Fc gamma receptors (Fc $\gamma \mathrm{Rs}$ ) are expressed on the CNS macrophages and bind to the constant domain of IgG, Activation of Fc $\gamma \mathrm{R}$ can result in a pro-inflammatory response including the release of cytokines and other mediators [28]. With respect to immunotherapy, anti-A $\beta$ antibodies will reach the CNS and will bind to and coat plaques which will promote the activation of microglia through Fc $\gamma$ Rs and thereby promote the phagocytosis of the antibody-coated plaques and release of pro-inflammatory mediators. These pro- inflammatory mediators are believed to be the cause of the vascular side effects that are observed post immunotherapy. Thus tackling the pro inflammatory response that occurs is a major challenge for the reduction of side effects. As the M1 phenotype of microglia seems to be producing the harmful effects during CNS autoimmune inflammation, most therapeutic ideas aim at either deactivating M1 phenotype microglia or turning them into M2 phenotype cells. There are two compounds that have been used in promoting this anti -inflammatory switch. The first being tuftsin. Tuftsin is a naturally occurring tetrapeptide (threonine-lysine-proline-arginine) that promotes phagocytic activity for cells of monocytic origin, such as microglia along with other effects like enhanced chemotaxis and antigen presentation. It has also been reported that tuftsin promotes an anti- inflammatory switch in the microglial cells. [29].Ghrelin is the other molecule that is reported to have significantly suppressed the production of proinflammatory cytokines in LPS-stimulated microglia and has thereby caused a suppression of Experimental Autoimmune Encephalomyelitis in the experiment performed. [30]

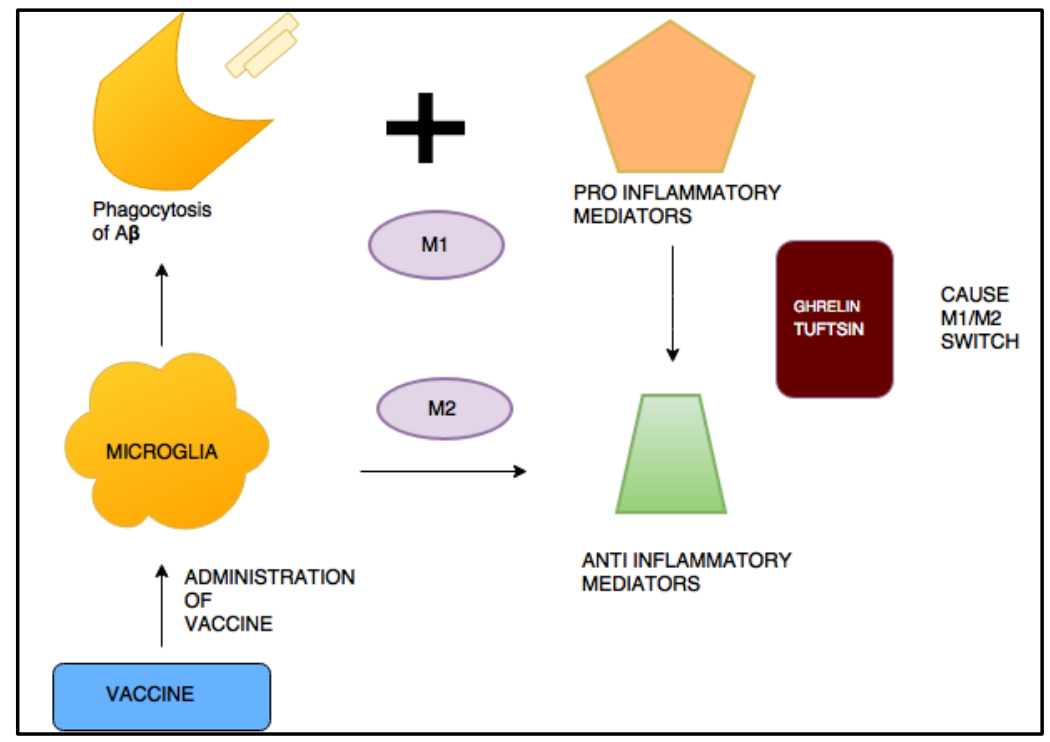


Fig. 3: Coupling immunotherapy with compounds like Ghrelin or Tuftsin that promotethe switch of M1 Phenotype to M2 Phenotype which causes the release ofAnti-inflammatory mediators.

\section{Conclusion}

While $A \beta$ is directly toxic to neurons, it can also lead to the enhancement of microglial neurotoxic effects by inducing inflammatory mediators. Degenerated neurons producemolecules other than $A \beta$ like glutamate and oxygen free radical species that enhance microglial neurotoxicity. However, the microglial neuroprotective effect resulting from $\mathrm{A} \beta$ clearance and antioxidant activity is obvious in $\mathrm{AD}$ and thus microglia cannot be completely deactivated. The conditions that determine microglial toxic or protective effects remain to be elucidated. Gaining more clarity on these issues may help to understand the AD pathophysiology in greater detail. A useful therapeutic strategy for $\mathrm{AD}$ may be to regulate and manipulate the microglia toward neuroprotection, specifically and $\mathrm{A} \beta$ clearance without inflammation. Initially, it was believed that inflammation was just a passive response to neuronal damage and degradation. However, increasing reports demonstrate that inflammation is capable of causing neuronal death actively and can exacerbate the damage that already exists which then fuels a self-propelling cycle of neuronal death. Immunotherapy is emerging as a choice of treatment, where an active vaccine can possibly trigger a process that uses the immune system to clear away the deposits of plaques of amyloid in the phagocytosis and release of pro inflammatory factors by the brain macrophages. However serious inflammation is caused due to the pro inflammatory factors that are released along with the phagocytosis process, Thus if the inflammatory aspect can be controlled on administering the vaccine, immunotherapy has a great scope. For this, we can use molecules like tuftsin and ghrelin that promote an antiinflammatory switch and promote the transition of the M1 phenotype to M2 phenotype and decrease the inflammatory environment. The vaccine will promote the clearance of the plaques by phagocytosis and molecules like tuftsin and ghrelin will promote the anti-inflammatory activity of the microglia thereby causing a reduction in the plaques along with attenuation of inflammation in totality. Thus, this study aims to highlight the importance of the balance that is maintained in the M1/M2 Macrophage Transition during normal healthy conditions which maintains a state of homeostasis. An imbalance in this state of polarization occurs in Alzheimer's disease which is caused by the hyper activation of the M1 Phenotype. This hyper activation then leads to a self-propelled cycle of excessive release of neurotoxic molecules by the microglia that cause neuronal death and further stimulation of the microglial cells due to molecules released by dying neurons like glutamate and reactive oxygen species, propelling this cycle. Thus, restoring the macrophage M1/M2 Phenotype balance serves as a potential therapeutic intervention in the treatment of Alzheimer's disease.

\section{References}

[1]. Saijo K, Glass CK (2011). Microglial cell origin and phenotypes in health and disease. Nature Reviews Immunology, 11 (11): 775 87 .

[2]. Zlokovic BV (2008). The blood brain barrier in health and chronic neurodegenerative disorders. Neuron ,57(2):178-201.

[3]. Lampron A, Elali A, Rivest S (2013). Innate Immunity in the CNS: Redefining the Relationship between the CNS and it's Environment. Neuron, 24;78 (2):214-32.

[4]. Jacobs S, Doering LC (2010) Astrocytes Prevent Abnormal Neuronal Development in the Fragile X Mouse. The Journal of Neuroscience, 24;30(12):4508-14.

[5]. Akiyama H (2000). Inflammation and Alzheimer's Disease. Neurobiology of Aging, 21(3):383-421.

[6]. Soulet D, Rivest S. (2008) Microglia. Current Biology, 18(12):R506-8.

[7]. Helmut Kettenmann (2013). Microglia: New Roles for the Synaptic Stripper. Neuron, Volume 77, Issue 1, Pages 10-18.

[8]. Amanda Sierra, Marie -Eye Tremblay (2014). Never Resting Microglia: Physiological Roles in the Healthy Brain and Pathological Implications. Frontiers in Cellular Neurosciece. $8: 240$

[9]. Tremblay ME (2011). The Role of Microglia in the Healthy Brain. The Journal of Neuroscience, 31(45):16064-9.

[10]. Boche D (2013). Review: Activation Patterns of Microglia and their Identification in the Human Brain. Neuropathology and Applied Neurobiology, 39(1):3-18.

[11]. Michael T. (2013). NLRP3 is activated in Alzheimer's disease and contributes to pathology in APP/PS 1 Mice. Nature, 493,674678.

[12]. Mosser DM, Edwards JP (2008). Exploring the Full Spectrum of Macrophage Activation. Nature Reviews Immunology, 8(12):95869.

[13]. Miyake Y (2007). Critical Role of Macrophages in the Marginal Zone in the Suppression of Immune Responses to Apoptotic CellAssociated Antigens. Journal of Clinical Investigation,117(8):2268-78.

[14]. Erin G.Reed- Geaghan (2011) Deletion of CD 14 Attenuates AD Pathology by Influencing the Brain's Inflammatory Milieu. Journal of Neuroscience, 30(46): 15369-15373.

[15]. SholpanAskarova (2011). Role of A $\beta$-Rage Interaction in Oxidative Stress and Cpla2 Activation in Astrocytes and Cerebral Endothelial Cells. Neuroscience, 199: 375-385.

[16]. EgleSolito (2012). Microglia Function in Alzheimer's Disease. Frontiers in Pharmacology. 3: 14.

[17]. Liu B, Hong JS. Role of Microglia in Inflammation - Mediated Neurodegenerative Diseases: Mechanisms and Strategies for Therapeutic Intervention. Journal of Pharmacology and Experimental Therapuetics. 304(1):1.

[18]. Wyss- Coray T (2006). Inflammation in Alzheimer Disease: Driving Force, By Stander or Beneficial Response ? Nature Medicine, 12(9):1005-15

[19]. Parkhurst CN (2013). Microglia Promote Learning - Dependent Synapse Formation Through Brain-Derived Neurotrophic Factor. Cell, 155(7):1596-609.

[20]. Salter MW (2014). Sublime Microglia: Expanding Roles For the Guardians of the CNS. Cell, 158(1):15-24.

[21]. Kettenmann H (2011). Physiology of Microglia. Physiological Reviews, 91(2):461-553. 
[22]. Squarzoni P (2014). Microglia Modulate Wiring of the Embryonic Forebrain. Cell Reports, 8(5):1271-9.

[23]. Walace Gomes-Leal (2012). Microglial Physiopathology: How to Explain the Dual Role of Microglia after Acute Neural Disorders? Brain Behaviour, 2(3): 345-356.

[24]. Shimohama S ( 2000). Apoptosis in Alzheimer's Disease- An Update. Apoptosis, 5(1):9-16.

[25]. . Barger SW (2001). Activation of Microglia by Secreted Amyloid Precursor Protein Evokes Release of Glutamate by Cystine Exchange and Attenuates Synaptic Function. Journal of Neurochemistry, 76(3):846-54.

[26]. Hideyuki Takeuchi (2005) Neuritic Beading Induced By Activated Microglia is an Early Feature of Neuronal Dysfunction Toward Neuronal Death by Inhibition of Mitochondrial Respiration and Axonal Transport. The Journal of Biological Chemistry. $280,10444-10454$.

[27]. Howard 1. Weiner, Dennis J. Selkoe (2002). Inflammation and Therapeutic Vaccination in CNS Diseases. Nature 420, 879-884.

[28]. James P. Fuller. (2014) New Roles for Fc Receptors in Neurodegeneration- The Impact on Immunotherapy for Alzheimer's Disease. Frontiers in Neuroscience, 8: 235.

[29]. Muzhou Wu (2012) Tuftsin Promotes an Anti- Inflammatory Switch and Attenuates Symptoms in Experimental Autoimmune Encephalomyelitis. Plos.7(4):e34933.

[30]. Michael Mark Theil (2009).Suppression of Experimental Autoimmune Encephalomyelitis by Ghrelin. The Journal of Immunology, 183(4):2859-66. 